\title{
Virtudes del médico: ¿qué importancia le atribuyen los pacientes?
}

\author{
SERGIO CARVAJAL ${ }^{1,2}$
}

\section{A survey among patients about physician virtues that they value}

Background: Theoretically, the exercise of good medicine requires physicians who possess and practice virtues. There are good reasons to believe that virtue ethics would be highly appreciated by patients. Aim: To determine the importance that patients attribute to the possession virtues among physicians. Material and Methods: Patients hospitalized in a private and a public hospital were invited to answer a three-question survey. The questions were: first, what do you expect of a good physician? Second, please evaluate, in a scale from 1 to 5 , the importance of physician virtues to consider him a good doctor (fidelity to trust given by the patient, benevolence, postponement of self-interests, compassion, intellectual honesty, justice and prudence). Third, among the seven former virtues, select the three more important, and then the most important of all. Results: Most patients responded that they valued that a good physician should have good communication skills, a cordial relationship, commitment to the patient and knowledge. All virtues studied were considered important or very important by almost all patients. The virtues considered the most important were intellectual honesty and fidelity to trust given by the patient. Conclusions: These results support the theoretical argument that, for patients, the practice of virtue ethics is essential for a good medical practice.

(Rev Med Chile 2020; 148: 1767-1772)

Key words: Ethics, Clinical; Ethics, Medical; Patient Preference; Physician-Patient Relations; Virtues.

$\mathrm{E}$ $\mathrm{n}$ la bioética clínica es ampliamente conocido el principialismo, basado en la propuesta de Beauchamp y Childress ${ }^{1}$. Estos autores establecen principios -beneficencia, no maleficencia, respeto por la autonomía, justicia- que deben guiar las actuaciones de los profesionales de la salud. Este enfoque deja en un plano secundario la importancia de las cualidades morales de la persona que actúa, en este caso el médico, pues se centra en la aplicación de principios y reglas. El principialismo está apoyado fundamentalmente en las teorías éticas deontológica y utilitarista, las cuales responden a la pregunta "¿qué debo hacer?" caso de la deontología se busca establecer normas racionales, idealmente universales, que indiquen cuales son los deberes a cumplir. En cambio, en
${ }^{1}$ Centro de Bioética, Facultad de Medicina Clínica AlemanaUniversidad del Desarrollo. Santiago, Chile.

${ }^{2}$ Comité de ética asistencial y unidad de gastroenterología, Clínica Santa María. Santiago, Chile.

Una versión extensa de este trabajo recibió el Premio de Ética 2019 otorgado por el Colegio Médico de Chile.

Trabajo no recibió financiamiento El autor declara no tener conflictos de interés.

Recibido el 5 de junio de 2020, aceptado el 12 de noviembre de 2020.

Correspondencia a: Dr. Sergio Carvajal C. Centro de Bioética Facultad de Medicina CAS-UDD Avenida Las Condes 12461 Edificio 3 Oficina 202, Las Condes, Santiago, Chile. scarvajal@udd.cl el utilitarismo la corrección de una acción está determinada por sus consecuencias, afirmando que lo correcto es hacer lo que proporcione mayor felicidad al mayor número de individuos ${ }^{3}$.

Existe una ética que presenta un enfoque y fundamentos diferentes a las anteriores, es la denominada ética de la virtud, la que está referida a las preguntas ¿qué tipo de persona debo llegar a ser? y ¿qué clase de vida debería llevar? ${ }^{2}$; es decir, su centro de atención es el agente moral, la persona que actúa. La teoría de la virtud fue desarrollada especialmente por Aristóteles y predominó durante muchos siglos en la filosofía moral. En griego virtud proviene de areté (excelencia), e implica la disposición del ánimo y el esfuerzo en la búsqueda de la excelencia ${ }^{4}$. Para Aristóteles la virtud es una 
manera habitual de actuar, fruto de una manera de ser, y que se ha trabajado con disciplina y esfuerzo para que se transforme en espontánea, para que llegue a ser un hábito ${ }^{5}$. Desde las últimas décadas del siglo XX la ética de la virtud ha sido revitalizada $y$ actualizada por varios autores ${ }^{6-8}$. Para MacIntyre una virtud es una cualidad humana adquirida, cuya posesión y ejercicio tiende a hacernos capaces de lograr aquellos bienes que son internos a las prácticas y cuya carencia nos impide lograr cualquiera de tales bienes ${ }^{8}$.

En la bioética clínica, diversos autores contemporáneos han resaltado que la posesión y práctica de virtudes por parte del médico es de alta importancia para una buena relación médico-paciente y una medicina de excelencia ${ }^{9-11}$. Los principales representantes del desarrollo de la ética de la virtud en el campo de la medicina han sido Pellegrino y Thomasma, quienes consideran que es posible llegar a un consenso acerca del bien y de las virtudes en el contexto de la ética profesional ${ }^{2}$, a partir de la naturaleza y propósito de la medicina, basada en el encuentro clínico. El florecimiento del profesional y la excelencia de su actividad no son posibles sólo por contratos, principios o cálculos de costo/beneficio, sino que se necesitan maneras de ser y modelos de ejercer bien la profesión, más allá de hacerlo de forma deontológica o contractualmente correcta ${ }^{4}$.

Pellegrino define la virtud como un rasgo del carácter que dispone a su poseedor a intentar, habitualmente, lograr la excelencia con respecto al telos (finalidad) específico de su actividad humana $^{12}$. Como el alivio del sufrimiento causado por enfermedades, el cuidado y la curación, el evitar muertes prematuras y buscar una muerte en paz, y la promoción de la salud son los fines de la medicina ${ }^{13}$, los rasgos de carácter que disponen a cumplirlos excelentemente constituyen las virtudes de las profesiones de la medicina. La posesión de estas virtudes define al buen médico. Pellegrino propone que las principales de estas virtudes son: fidelidad a la confianza entregada por el paciente (el médico debe mantenerse fiel a la confianza que el paciente deposita en él), benevolencia, postergación del interés propio del profesional, compasión y cuidado, honestidad intelectual (reconocer cuando no se sabe algo y admitirlo), justicia y prudencia ${ }^{12}$.

Se estima que la posesión de virtudes por parte del médico es indispensable para ejercer una buena medicina ${ }^{14-18}$, centrada en el bien del paciente como persona. Se recalca la importancia de la adquisición de virtudes durante la formación de los médicos ${ }^{19-21}$, las que pueden ser aprendidas especialmente mediante modelos a seguir ${ }^{22}$. Así entonces, son muy razonables los argumentos respecto a la importancia que el médico posea las virtudes propias de su profesión, sin embargo, existe escasa investigación que busque conocer la apreciación y opinión de los pacientes en este aspecto. Los pocos datos existentes relacionados al tema apuntan en la misma dirección que la argumentación teórica ${ }^{23-27}$.

Considerando lo expuesto, resulta atrayente averiguar cuán importante es para los pacientes que el médico posea virtudes. El objetivo general de este trabajo es determinar la importancia que los pacientes atribuyen a la posesión de virtudes por parte médico. Los objetivos específicos son: establecer qué cualidades del médico afloran espontáneamente en los pacientes al preguntarles por las características de un buen médico; establecer el grado de importancia que los pacientes atribuyen a la posesión de ciertas virtudes por parte del médico y establecer el grado de importancia relativa que los pacientes atribuyen a las diferentes virtudes del médico.

\section{Pacientes y Método}

Se diseñó un estudio observacional de corte transversal, consistente en entrevistar a 100 pacientes hospitalizados, 50 en una institución de salud privada (Clínica Santa María) y 50 en un hospital del sistema público (Hospital Padre Hurtado), aplicando una encuesta de tres preguntas. El criterio de inclusión fue pacientes adultos, entre 18 y 80 años de edad, hospitalizados por patología médica o quirúrgica, que se encontraran en un estado de conciencia normal. Los pacientes entrevistados fueron aquellos disponibles los días en que el entrevistador concurrió a los centros de salud. No se accedió a la ficha clínica y sólo se consignó la información que cada paciente entregó en forma voluntaria. No se registró en la encuesta datos que permitieran identificar al paciente posteriormente. El entrevistador fue el autor del estudio, quien no era parte de los médicos que atendían a los pacientes. Se obtuvo el consentimiento informado de cada paciente antes de entrevistarlo. El estudio fue aprobado por los comités de ética científica de 
la Facultad de Medicina Clínica Alemana-Universidad del Desarrollo y de la Clínica Santa María.

La encuesta consistió en tres preguntas. La pregunta 1, una pregunta abierta, en que se consultó qué es lo que espera el paciente de un buen médico. En la pregunta 2 se pidió al paciente que calificara, en una escala de 1 a 5, la importancia de cada una de las cualidades que se le nombraban de un médico para considerarlo un buen médico. Una cualidad correspondió a conocimientos y habilidad técnica y las otras a las virtudes propuestas por Pellegrino como las más importantes virtudes del médico ${ }^{12}$. A cada paciente se le facilitó un glosario aclarando el significado de las virtudes en un lenguaje comprensible y, en caso necesario, se le explicó verbalmente. En la pregunta 3 se le presentó al paciente una hoja con un listado de las mismas virtudes (cuyo orden en la lista fue sorteado), para que consignara cuáles de ellas consideraba las tres más importantes, asignándoles primero, segundo y tercer lugar.

\section{Resultados}

Se contactó a 104 pacientes, 4 de los cuales declinaron participar. De acuerdo a lo planificado, la muestra quedó constituida por 100 pacientes hospitalizados (50 en el Hospital Padre Hurtado y 50 en la Clínica Santa María). El rango de edad del grupo es de 18 a 80 años, con un promedio de $54 \pm 17$ años; 57 hombres y 43 mujeres.

\section{Resultados pregunta 1}

A esta pregunta abierta (¿qué es lo que Ud. espera de un buen médico?), la mayoría de los pacientes respondió expresando uno o dos conceptos. Se agruparon los conceptos en cuatro categorías, las que fueron creadas sólo después de analizar las respuestas obtenidas, es decir, no se diseñaron antes de las entrevistas. Las categorías son las siguientes:

A) Que el médico establezca una buena comunicación con el paciente, informándolo con explicaciones claras, entendibles y que sea franco en dicha información. Ejemplos de respuestas son: "que sea claro en las explicaciones", "que explique bien", "que tenga buena comunicación", "que mantenga informado", "que explique en lenguaje no técnico", "que me informe de lo bueno y lo malo", "que sea sincero" y "que sea franco en la información".
B) Que el médico establezca una buena relación con el paciente, con simpatía, cercanía, amabilidad y cordialidad. Ejemplos de respuestas son: "que tenga un trato cordial", "que sea amable", "que atienda bien", "buen trato", "que tenga paciencia", "que sea humano, que trate con cariño", "que sea acogedor", "gentileza", "que sea humilde, atento" y "que tenga calidez".

C) Que el médico tenga y demuestre real preocupación por el paciente, que lo conozca y que se pueda poner en su lugar. Ejemplos de respuestas son: "que se preocupe por mi caso", "que conozca al paciente realmente", "que se ponga en el lugar del paciente", "que sea más amigo del paciente", "preocupación por mi problema", "interés por el paciente", "que me escuche" y "que sea fiel".

D) Que el médico tenga competencia técnica, que posea buen nivel de conocimientos, que su diagnóstico y tratamiento sean correctos y oportunos, inspirando seguridad. Ejemplos de respuestas son: "atención rápida y efectiva", "por supuesto que conocimientos", "un buen diagnóstico", "competencia profesional", "que tenga seguridad y experiencia", "que me mejore", "que tenga competencias técnicas", "diagnóstico certero", "buenos resultados", "que sepa lo que hace", "que solucione el problema" y "que haga bien su pega".

El porcentaje de pacientes que mencionaron cada categoría se aprecia en la Tabla 1.

\section{Resultados pregunta 2}

En esta pregunta, cada paciente asignó una nota, de 1 hasta 5 , a la importancia que le atribuyó a cualidades que un médico debe poseer para considerar que es un buen médico. La escala de notas es: 1: no es importante, 2: poco importante, 3: medianamente importante, 4: importante, 5: muy importante. Las ocho cualidades evaluadas corresponden a las siete virtudes estudiadas $y$, la octava, al conocimiento y competencia técnica del médico.

El resultado obtenido en esta pregunta queda bien expuesto señalando el porcentaje de pacientes que estima que la virtud del médico consultada es importante o muy importante (nota 4 o 5), lo que se muestra en la Tabla 2. 
Tabla 1. Porcentaje de menciones por categoría

\begin{tabular}{|c|c|}
\hline $\begin{array}{l}\text { ¿Qué espera usted de un bu } \\
\text { Categorías }\end{array}$ & $\begin{array}{l}\text { nédico? } \\
\% \text { de } \\
\text { pacientes }\end{array}$ \\
\hline $\begin{array}{l}\text { Categoría A: } \\
\text { Buena comunicación, explicación clara } \\
\text { y franca }\end{array}$ & $38 \%$ \\
\hline $\begin{array}{l}\text { Categoría B: } \\
\text { Buena relación, amabilidad, cordialidad } \\
\text { y cercanía }\end{array}$ & $45 \%$ \\
\hline $\begin{array}{l}\text { Categoría C: } \\
\text { Preocupación por el paciente, conocer } \\
\text { al paciente }\end{array}$ & $34 \%$ \\
\hline $\begin{array}{l}\text { Categoría D: } \\
\text { Conocimientos y competencia técnica }\end{array}$ & $46 \%$ \\
\hline
\end{tabular}

Nota: el total suma más de 100 pues cada paciente respondió uno o más conceptos.

Respecto al conocimiento y competencia técnica del médico, todos los pacientes consideraron que es importante o muy importante.

\section{Resultados pregunta 3}

Se obtuvo respuesta de 99 pacientes, cada uno de los cuales escogió las tres virtudes que consideró más importantes de las siete virtudes consultadas. Además las ordenó en primer, segundo y tercer lugar. No se obtuvo respuesta válida de un pacien-

Tabla 3. Cantidad de pacientes que eligieron a la virtud que se indica entre las tres más importantes

\begin{tabular}{|lc|}
\hline Virtud & n de pacientes (\%) \\
\hline Honestidad intelectual & $83(83,8 \%)$ \\
\hline Fidelidad a la confianza & $65(65,7 \%)$ \\
\hline Benevolencia & $40(40,4 \%)$ \\
\hline Prudencia & $40(40,4 \%)$ \\
\hline Compasión & $38(38,4 \%)$ \\
\hline Justicia & $16(16,2 \%)$ \\
\hline Postergación del interés personal & $15(15,2 \%)$ \\
\hline
\end{tabular}

Nota: el total de pacientes en esta respuesta es 99. Un paciente no respondió por considerar que todas las virtudes tienen la misma importancia.
Tabla 2. Porcentaje de pacientes que estiman que la virtud del médico consultada es importante o muy importante

\begin{tabular}{|lc|}
\hline Virtud & $\begin{array}{c}\text { \% de pacientes } \\
\text { (n = 100) }\end{array}$ \\
\hline Fidelidad a la confianza & $98 \%$ \\
Benevolencia & $98 \%$ \\
\hline Compasión & $91 \%$ \\
\hline Honestidad intelectual & $100 \%$ \\
\hline Postergación del interés personal & $88 \%$ \\
\hline Prudencia & $99 \%$ \\
\hline Justicia & $92 \%$ \\
\hline Conocimiento y habilidad técnica* & $100 \%$ \\
\hline
\end{tabular}

*Cualidad incluida en la encuesta, no pertenece a las virtudes.

te, pues consideró que todas las virtudes tienen la misma importancia.

La honestidad intelectual y la fidelidad a la confianza aparecen como las virtudes más importantes en la elección de los pacientes. La cantidad de pacientes que indicaron que determinada virtud está entre las tres más importantes se muestra en la Tabla 3. Los resultados respecto a la virtud considerada en el primer lugar, como la más importante, se muestra en la Tabla 4.

Tabla 4. Virtud del médico elegida como la más importante por los pacientes

\begin{tabular}{|lc|}
\hline Virtud & $\begin{array}{c}\text { Pacientes } \\
\text { n (\%) }\end{array}$ \\
\hline Honestidad intelectual & $30(30,3 \%)$ \\
\hline Fidelidad a la confianza & $29(29,3 \%)$ \\
\hline Compasión & $13(13,1 \%)$ \\
\hline Benevolencia & $9(9,1 \%)$ \\
\hline Prudencia & $7(7,1 \%)$ \\
\hline Justicia & $7(7,1 \%)$ \\
\hline Postergación del interés personal & $4(4 \%)$ \\
\hline
\end{tabular}

Nota: el total de pacientes en esta respuesta es 99. Un paciente no respondió por considerar que todas las virtudes tienen la misma importancia. 


\section{Discusión}

La conclusión central del estudio es que los pacientes entrevistados estiman que para ser un buen médico es relevante poseer las virtudes de la profesión. Desde su experiencia como pacientes, las personas piensan que las virtudes del médico son importantes o muy importantes para el ejercicio de una buena medicina por parte del profesional.

Ante la pregunta abierta respecto a lo que espera de un buen médico, la mayoría de los pacientes consideran aspectos relacionados con características personales y virtudes del médico, mientras que menos de la mitad se refirieron espontáneamente a los aspectos técnicos. Por cierto que no existe una contraposición entre lo técnico y lo "humano" en el profesional, sino que por el contrario, lo deseable es que ambas dimensiones sean de excelencia. Debe recalcarse que para los pacientes la dimensión "humana" o personal del médico es de alta importancia.

Respecto a la estimación de la importancia que tienen las virtudes en un médico, los resultados muestran que la amplia mayoría de pacientes entrevistados juzgan que ellas son muy relevantes. $\mathrm{Al}$ analizar el porcentaje de pacientes que asignó nota 4 o 5 a cada virtud (es decir importante o muy importante), casi todas alcanzaron entre 90 y $100 \%$. Sólo la postergación del interés personal del médico obtuvo menos de $90 \%$ (88\%). Hay que destacar que la cualidad de conocimiento y habilidad técnica fue considerada importante o muy importante por el $100 \%$ de los pacientes, lo que refuerza que los pacientes consideran que el profesional debe dominar los aspectos técnicos y a la vez practicar las virtudes que se esperan de él. Se espera un médico integral, un "buen médico bueno".

Es interesante notar que al preguntar por cuáles de las virtudes son más importantes, la honestidad intelectual es considerada en el primer lugar, seguida muy de cerca por la fidelidad a la confianza. Mientras que la justicia y la postergación del interés personal del médico quedaron bastante atrás en las preferencias. Por tanto, las virtudes con más importancia para los pacientes son aquellas que apuntan a la confiabilidad del médico.

Los resultados obtenidos en este estudio son coincidentes con las argumentaciones teóricas en el sentido que las virtudes del médico y la práctica de la ética de la virtud son requisitos indispensa- bles para una excelencia de los profesionales y para el ejercicio de una buena medicina. Por tanto, es altamente deseable que la adquisición de virtudes por parte de los estudiantes de medicina sea una preocupación preferente de las escuelas de medicina y un eje curricular a lo largo de la enseñanza de pre y postgrado. Es necesario que los docentes comprendan que las virtudes forman parte importante de lo que deben transmitir y enseñar en la práctica diaria, puesto que ellas no se aprenden en la sala de clases sino que se adquieren con el ejemplo y la observación crítica.

Es necesario señalar las limitaciones de esta investigación. En primer término se debe aclarar que los resultados obtenidos reflejan la opinión de los pacientes entrevistados y ello no es generalizable con propiedad al universo de pacientes chilenos, pues para ello habría sido necesario aplicar técnicas de muestreo de alta complejidad. Sin embargo, a pesar de esta limitación, se debe destacar que se obtuvo la opinión de un centenar de pacientes de nuestro medio y provenientes de ambos sistemas de salud, por lo que los resultados son válidos como una manera de afirmar con información real lo planteado en la teoría. En segundo lugar, hay que consignar que se seleccionó algunas de las virtudes del médico, existiendo varias más. Las elegidas fueron las que señala Pellegrino como las más significativas, y representan el núcleo de las virtudes del médico.

En síntesis, este estudio que, de acuerdo a lo investigado, es el primero en su tipo en nuestro país, muestra que para los pacientes es de alta importancia que el médico posea las virtudes de su profesión para ser considerado un buen médico. Por tanto esto debe ser considerado como de primera jerarquía en la formación y educación contínua de los médicos.

Agradecimientos: Al doctor Juan Pablo Beca, por su valiosa guía y consejos para desarrollar este trabajo. A los pacientes que participaron en este estudio.

\section{Referencias}

1. Beauchamp TL, Childress JF. Principles of Biomedical Ethics. 5th ed. New York: Oxford University Press; 2001.

2. Cruz J. Bioética y teorías de la virtud. En: Ferrer JJ, Leca- 
ros JA, Molins Mota R, editores. Bioética: el pluralismo de la fundamentación. Madrid: Universidad Pontificia Comillas; 2016. p. 175-204.

3. Misselbrook D. Virtue ethics - and old answer to a new dilemma? Part 1. Problems with contemporary medical ethics. J R Soc Med 2015; 108 (2): 53-6.

4. Román B. Etica de la virtud para la bioética clínica: hábitos, excelencia y conciencia de pertenencia. En: Beca JP, Astete C, editores. Bioética Clínica. Editorial Mediterráneo; 2012. p. 62-74.

5. Aristóteles. Etica Nicomaquea. 23 ed. México,DF: Editorial Porrúa; 2013.

6. Díaz García A. La ética de la virtud y la bioética. Rev Colomb Bioética 2009; 4 (1): 93-128.

7. Anscombe G. Modern Moral Philosophy. Philosophy 1958; 33 (124): 1-16.

8. MacIntyre A. Tras la virtud. Segunda ed. Barcelona: Critica; 2004.

9. Pellegrino ED, Thomasma DC. The Virtues in Medical Practice. New York: Oxford University Press; 1993.

10. Toon PD. A Flourishing Practice? 1st ed. London: Royal College of General Practitioners; 2014.

11. Oakley J, Cocking D. Virtue Ethics and professional Roles. 1st ed. Cambridge, UK: Cambridge University Press; 2004.

12. Pellegrino ED. Toward a virtue-based normative ethics for the health professions. Kennedy Inst Ethics J 1995; 5 (3): 253-77.

13. Hastings Center. Los fines de la medicina. Cuaderno11 ed. Fundació Victor Grífols i Lucas; 2007.

14. Toro A. Consideraciones acerca de las cualidades del buen médico y del profesionalismo en medicina. Acta Médica Colomb 2011; 36 (1): 45-50.

15. Bryan CS, Babelay AM. Building Character: A Model for Reflective Practice. Acad Med 2009; 84 (9): 1283-8.
16. Coverdale JH, Franzcp RB, Roberts LW. Cultivating the professional virtues in medical training and practice. Acad Psychiatry 2011; 35 (3): 155-9.

17. Mitchell D. Philosophy at the bedside - Phenomenology, complexity and virtue in the care of patients. J Eval Clin Pract 2014; 20 (6): 970-4.

18. Siegler $\mathrm{M}$ a. Professional values in modern clinical practice. Hastings Cent Rep 1991; 30 (4 Suppl): S19-22.

19. López L, Dyck AJ. Educating physicians for moral excellence in the twenty-first century. J Relig Ethics 2009; 37 (4): 651-68.

20. Toledo-Pereyra LH. Humility. J Investig Surg 2007; 20 (2): 63-5.

21. Vidal-Gual J. Las virtudes en la medicina clínica. Arch en Med Fam 2006; 8 (1): 41-52.

22. Pellegrino ED. Professionalism, profession and the virtues of the good physician. Vol. 69, The Mount Sinai journal of medicine, New York. 2002. p. 378-84.

23. macmillan.org [Internet]. [consultado 2016 Feb 26]. Disponible en: http://www.macmillan.org.uk/Aboutus/ News/Latest_News/BritonsbelievebeingtreatedwithdignityinNHSasimportantasgettingbesttreatment.aspx

24. Ortona C, Fortes P. Journalists want virtuous physicians?: An analysis by the model of Ethic of Virtues, in reference to Beneficence, according to Pellegrino and Thomasma. Acta Bioeth 2015; 21 (1): 109-16.

25. Bendapudi NM, Berry LL, Frey K a, Parish JT, Rayburn WL. Patients' perspectives on ideal physician behaviors. Mayo Clin Proc 2006; 81 (3): 338-44.

26. Motilla M, Gómez RS, Curiel O. ¿ Qué cualidades valoran más los Pacientes de su Médico de Familia ? 2009; 2 (6): 263-8.

27. Oseguera-Rodríguez J, Viniegra-Velásquez L. Características humanistas del médico deseadas por la sociedad. Rev Med Inst Mex Seguro Soc 2008; 46 (2): 171-8. 